\title{
Qualidade de vida entre enfermeiros e relação com o nível de complexidade do trabalho em saúde
}

\section{Quality of life among nurses and relationship with the level of complexity of health work}

\author{
Melina Lopes Lima \\ Universidade Estadual de Ponta Grossa - UEPG - Ponta Grossa - Brasil \\ enfmelina@gmail.com \\ Luciane Patricia Andreani Cabral \\ Universidade Estadual de Ponta Grossa - UEPG - Ponta Grossa - Brasil \\ luciane.pacabral@gmail.com \\ Danielle Bordin \\ Universidade Estadual de Ponta Grossa - UEPG - Ponta Grossa - Brasil \\ daniellebordin@hotmail.com \\ Bruno Pedroso \\ Universidade Estadual de Ponta Grossa - UEPG - Ponta Grossa - Brasil \\ prof.brunopedroso@gmail.com \\ Caio Muiños Parrode de Godoy \\ Secretaria de Saúde do Paraná - Ponta Grossa - Brasil \\ caiofloresta@gmail.com \\ Cristina Berger Fadel \\ Universidade Estadual de Ponta Grossa - UEPG - Ponta Grossa - Brasil \\ cbfadel@gmail.com
}

\section{Resumo}

A Qualidade de Vida no Trabalho (QVT) é definida como o grau de satisfação de suas expectativas pessoais, através de experiências no trabalho, enquanto atinge objetivos organizacionais. No contexto dos profissionais da saúde, a área da enfermagem integra uma das parcelas de trabalhadores que está cotidianamente exposta a demandas e exigências psicobiológicas do processo de trabalho. Com relação ao processo de trabalho de enfermeiros em diferentes modelos de atenção à saúde, pretende-se analisar a qualidade de vida de profissionais enfermeiros e relacioná-la com a complexidade do trabalho em saúde. Estudo transversal, tipo inquérito, com a utilização de metodologia quantitativa, de caráter exploratório e descritivo, desenvolvido junto a enfermeiros da atenção primária $(n=52)$ e terciária $(n=47)$ à saúde do município de Ponta Grossa - PR. Para a coleta de dados utilizou-se o instrumento WHOQOL-BREF. Não houve diferença $(p>0,05)$ na QV dos enfermeiros da atenção primária e terciária. Não teve diferença $(p>0,05)$ na QV em relação à idade, gênero e estado civil. Houve uma maior diferença de médias no domínio meio ambiente, sendo maior no grupo de enfermeiros da atenção primária à saúde (APS) e pior no hospital universitário (HU). O presente estudo 
possibilitou analisar e conhecer os pontos de fragilidade na QV de enfermeiros que atuam na APS e em um HU, e fomentou questões importantes para a compreensão do contexto de trabalho do enfermeiro.

Palavras-chave: qualidade de vida, níveis de atenção à saúde, profissionais de enfermagem.

\begin{abstract}
Quality of Life at Work (QWL) is defined as the degree of satisfaction of your personal expectations, through experiences at work, while achieving organizational goals. In the context of health professionals, the nursing area integrates one of the plots of workers who are daily exposed to the psychobiological demands and demands of the work process. Regarding the work process of nurses in different models of health care, it is intended to analyze the quality of life of professional nurses and relate it to the complexity of health work. Cross-sectional, survey-type study, using quantitative, exploratory and descriptive methodology, developed with primary care $(n=52)$ and tertiary $(n=47)$ health nurses in the municipality of Ponta Grossa - PR. For data collection, the WHOQOL-BREF instrument was used. There was no difference $(p>0.05)$ in the QOL of nurses in primary and tertiary care. There was no difference $(p>0.05)$ in QOL in relation to age, gender and marital status. There was a greater difference of means in the environment domain, being greater in the group of nurses in primary health care (PHC) and worse in the university hospital $(\mathrm{HU})$. The present study made it possible to analyze and understand the weaknesses in the QoL of nurses working in $\mathrm{PHC}$ and in a $\mathrm{HU}$ and promoted important questions for understanding the nurses' work context.
\end{abstract}

Keywords: quality of life, levels of health care, nursing professionals.

\title{
1. Introdução
}

A primeira publicação sobre qualidade de vida (QV) foi consolidada em 1969, pela revista norte americana Science (STEPHENS, 1969), e desde então o tema tem sido vastamente explorado, ainda que não exista consenso na literatura quanto a sua concepção. Em 1995, a Organização Mundial de Saúde (OMS) reuniu especialistas em todo o mundo e imprimiu o seguinte conceito:

Qualidade de vida é a percepção do indivíduo de sua posição na vida no contexto da cultura e sistema de valores nos quais ele vive e em relação aos seus objetivos, expectativas, padrões e preocupações (THE WHOQOL GROUP, 1995).

No campo do trabalho, a qualidade de vida é definida como a satisfação do trabalhador com o seu trabalho, sendo esta afetada por sentimentos e percepções individuais (MOSADEGHRAD et al., 2011). Em especial para o profissional enfermeiro, a QV no trabalho é definida como o grau de satisfação de suas expectativas pessoais, através de experiências no trabalho, enquanto atinge objetivos organizacionais (BROOKS; ANDERSON, 2005).

No contexto dos profissionais da saúde, a área da enfermagem integra uma das parcelas de trabalhadores que está cotidianamente exposta a demandas e exigências psicobiológicas do processo de trabalho, as quais geram, ao longo do tempo, desgaste das capacidades vitais do trabalhador (BECK et al., 2006). Diversos estudos associam a qualidade de vida no trabalho do enfermeiro a índices baixos de satisfação em variados países (ALMALKI et al., 2012; BROOKS et al., 2007; FATHIMATH et al., 2014; BATTU; CHAKRAVARTHY, 2014; AKTER et al., 2017; PERRY et al., 2017).

Muitas vezes o trabalho cotidiano do enfermeiro envolve situações como morte, conflitos com médicos e outros profissionais, carga horária excessiva de trabalho, 
problemas com pacientes e familiares e ainda, práticas discriminatórias com a profissão (AKTER et al., 2017; SILVA et al., 2017), as quais podem se desdobrar em agentes estressores para esses sujeitos. O estresse ao qual o enfermeiro é submetido pode acarretar em absenteísmo (FU et al., 2015), problemas de saúde (SUN et al., 2017), acidentes de trabalho, doenças do sono, dor (PERRY et al.,2017), fadiga (JANG et al., 2016), redução na qualidade de vida (FU et al.,2015; AKTER et al.,2017; SILVA et al., 2017) e intenção de abdicar da profissão (CHOl et al., 2017; FU et al., 2015).

Em especial no âmbito hospitalar, o trabalho do enfermeiro é exigente, complexo e repleto de particularidades, tais como a organização de seu processo de trabalho em turnos ininterruptos, o estabelecimento de relações interpessoais com colegas, 0 cumprimento de regimentos, normas e rotinas preestabelecidas em cada área de atuação, a divisão fragmentada de tarefas, a rígida estrutura hierárquica e o dimensionamento insuficiente de pessoal (QUEIROZ; SOUZA, 2012).

Por outro lado, profissionais que atuam em modelo distinto de atenção à saúde, a atenção primária, de menor nível de complexidade e cujos trabalhadores estão dispostos predominantemente em unidades de saúde, se deparam com especificidades distintas no trabalho, cujas características são: valorização e reconhecimento profissional, educação permanente, vínculo estabelecido com a comunidade, trabalho conjunto com todos integrantes da equipe, falta de recursos humanos, materiais e equipamentos, falta de compreensão e paciência da comunidade e dificuldades de relacionamento com os gestores. Tais características emergem nos enfermeiros sentimentos ambíguos com relação ao trabalho (SCHRADER et al., 2012).

Com relação ao processo de trabalho de enfermeiros em diferentes modelos de atenção à saúde, estudos demonstram relação elevada com agravos psicológicos e com impactos negativos na qualidade de vida, tanto entre profissionais da atenção primária a saúde (APS) quanto entre profissionais de setores hospitalares, com leve ampliação para profissionais da atenção terciária (HOLMES et al., 2014; ROSSI et al., 2010).

Cientes de que o reconhecimento da qualidade de vida de enfermeiros em seus diferentes níveis de complexidade no trabalho subsidia estratégias de melhoria a saúde destes profissionais, podendo impactar positivamente na qualidade dos serviços prestados à população, o objetivo do presente trabalho é analisar a qualidade de vida de profissionais enfermeiros e relacioná-la com a complexidade do trabalho em saúde.

\section{Método}

Realizou-se estudo exploratório transversal, tipo inquérito, com a utilização de metodologia quantitativa, de caráter descritivo e inferencial.

$\mathrm{O}$ estudo foi desenvolvido junto à totalidade de enfermeiros de um Hospital Universitário (HU) $(n=100)$, bem como com a totalidade de enfermeiros da Atenção Primária à Saúde (APS) ( $\mathrm{n=80}$ ), ambos do município de Ponta Grossa, estado do Paraná, Brasil, que se dispuseram livremente a participar do estudo. $O$ ano-base para a consulta foi 2018.

A coleta de dados foi realizada por meio de questionário com dados demográficos (sexo, idade e estado civil) e do questionário WHOQOL-BREF, versão abreviada em português do Instrumento de Avaliação de Qualidade de Vida da Organização Mundial de Saúde, validado por Fleck (2000). Este instrumento contém 26 questões, distribuídas em quatro domínios: relações sociais, psicológico, físico e meio ambiente. Cada domínio é composto por questões cujas pontuações de respostas variam entre 1 e 5.

Os dados de qualidade de vida foram tratados de acordo com o preconizado pela Organização Mundial da Saúde, detalhado por Pedroso et al. (2009), para execução em Microsoft Excel. 
Teste de normalidade (Kolmogorov-Smirnov) foi empregado a fim de definir a utilização de análise paramétrica ou não paramétrica. Os dados dos domínios físico, psicológico, relações sociais e meio ambiente apresentaram distribuição normal. Deste modo, aplicou-se o teste paramétrico $t$ de Student não pareado para avaliar estes domínios, segundo o local de atuação (HU x APS) e variáveis demográficas (sexo e estado civil). As médias da qualidade de vida geral não passaram no teste de normalidade, sendo então utilizado o teste $U$ de Mann-Whitney para comparação dos grupos HU x APS. Para a verificação dos domínios versos grupos etários $(\leq 30$ anos, $30>40$ anos e $\geq 40$ ) utilizou-se o teste ANOVA, pelo emprego de três grupos distintos. Todos os dados foram avaliados estatisticamente pelo programa Graphy Pad In Stat. (GRAPHPAD SOFTWARE, 2018).

Todos os participantes foram previamente informados sobre os objetivos da pesquisa, seu caráter de voluntariedade e de não-identificação, assim como sobre a forma de coleta, análise e destino dos dados. Os que aquiesceram com sua participação, o fizeram, inicialmente mediante o preenchimento de um Termo de Consentimento Livre e Esclarecido. O estudo foi aprovado pelo Comitê de Ética em Pesquisas com seres humanos da Universidade Estadual de Ponta Grossa (paracer n ${ }^{\circ}$ 2.146.155/2017 e parecer no 2.461.494/2018), respeitando os ditames da resolução 466/12 do Conselho Nacional de Saúde.

\section{Resultados}

Do total de 180 profissionais enfermeiros investigados, considerando ambos os grupos, 99 sujeitos responderam efetivamente aos questionários, sendo 47 do $\mathrm{HU}$ e 52 da APS. A média etária geral da amostra foi de 36,2 anos (23-62 anos; $\sigma=9,24)$, sendo composta, em sua maioria, por mulheres (84,85\%). Quanto ao estado civil, $56,57 \%$ dos participantes eram casados, $35,35 \%$ solteiros, e $8,08 \%$ divorciados.

Quanto à distribuição demográfica por local de atuação profissional, 47 (47,47\%) dos enfermeiros pertenciam ao HU e 52 (52,52\%) à APS. A média de idade de enfermeiros do hospital foi de 34,5 anos (23-62 anos; $\sigma=10,17)$ e da atenção básica, 37,8 anos (25-62 anos; $\sigma=8,12)$. Quanto ao estado civil, 12 enfermeiros hospitalares eram casados (25,53\%), 35 solteiros $(74,46 \%)$ e 1 divorciado (2,12\%). Na APS, 33 eram casados $(63,46 \%), 12$ solteiros $(23,07 \%)$ e 7 divorciados (13,46\%). Quanto ao gênero, predominância de mulheres em ambos os espaços: $78,72 \%$ no HU e 90,38\% na APS.

O Gráfico 1 representa os resultados da QV global e seus domínios formadores entre enfermeiros do HU e APS. 
Gráfico 1: Média e desvio padrão da Qualidade de Vida, segundo domínios formadores. Profissionais enfermeiros do HU e da APS

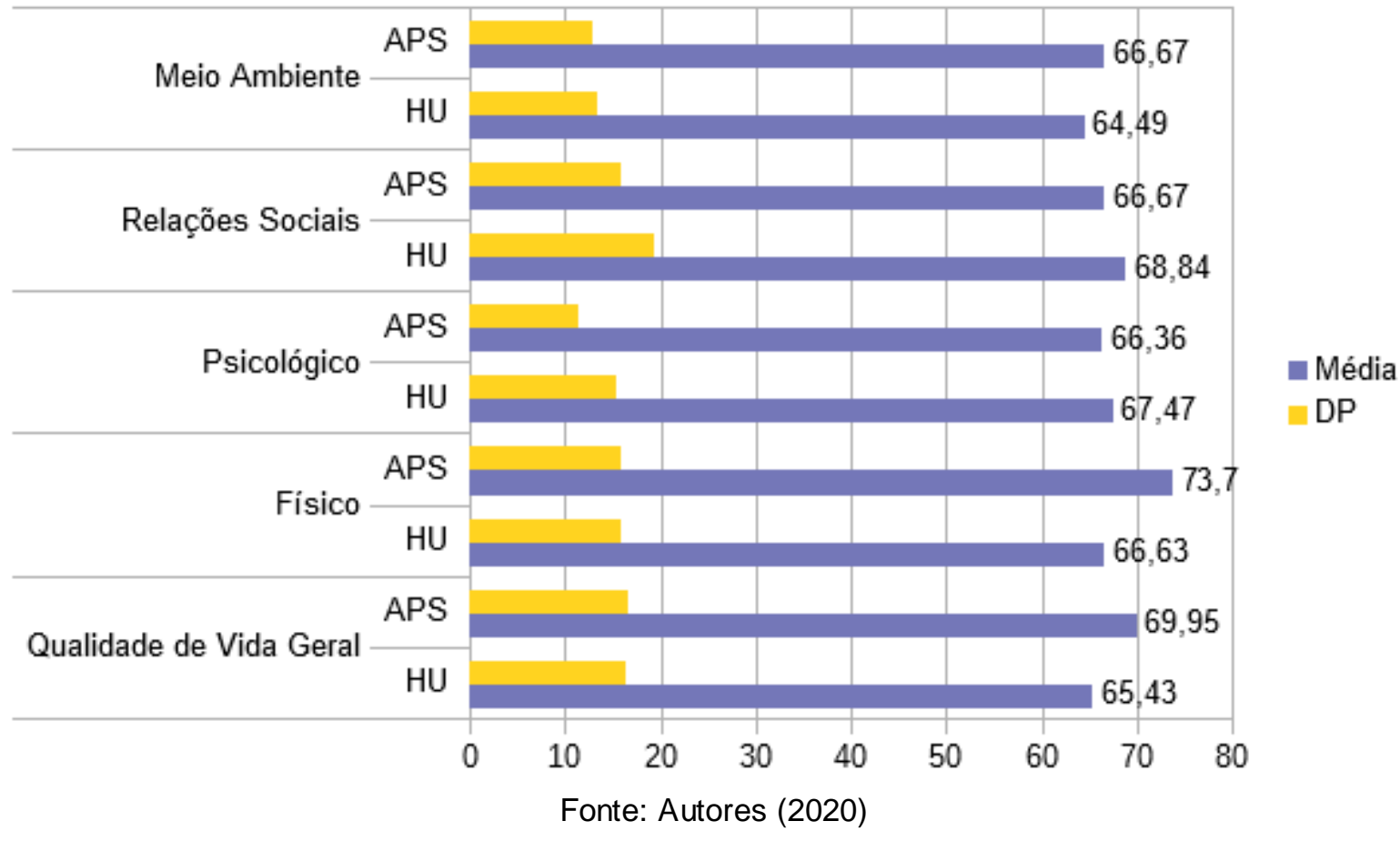

Em todos os domínios investigados os escores de QV de ambos os grupos foram semelhantes, não havendo diferença significativa entre eles $(p>0,05)$, no entanto, houve uma maior diferença de médias no domínio físico (HU: 66,6 x APS: 73,7). O domínio de maior escore foi no campo físico, entre enfermeiros da APS $(73,7)$ e o de menor escore no campo do meio ambiente, entre enfermeiros do HU $(64,5)$.

A Tabela 1 apresenta os domínios de qualidade de vida e QV Global segundo as variáveis demográficas dos enfermeiros de Ponta Grossa - PR da APS e HU.

Tabela 1: Média, desvio padrão (DP) e valor de $p$, de condições demográficas, segundo domínios de qualidade de vida de enfermeiros de Ponta Grossa-PR da APS e HU ( $n=99)$

\begin{tabular}{|c|c|c|c|c|c|c|c|c|c|c|}
\hline \multirow{2}{*}{$\begin{array}{c}\text { Variável } \\
\text { Demográ- } \\
\text { fica }\end{array}$} & \multicolumn{2}{|c|}{ Físico } & \multicolumn{2}{|c|}{ Psicológico } & \multicolumn{2}{|c|}{$\begin{array}{c}\text { Relações } \\
\text { Sociais }\end{array}$} & \multicolumn{2}{|c|}{ Meio Ambiente } & \multicolumn{2}{|c|}{ QV Global } \\
\hline & $\begin{array}{l}\text { Média } \\
\text { (DP) }\end{array}$ & $\begin{array}{c}\mathbf{p} \\
\text { valor }\end{array}$ & $\begin{array}{l}\text { Média } \\
\text { (DP) }\end{array}$ & $\begin{array}{c}\mathbf{p} \\
\text { valor }\end{array}$ & $\begin{array}{l}\text { Média } \\
\text { (DP) }\end{array}$ & $\begin{array}{c}p \\
\text { valor }\end{array}$ & $\begin{array}{l}\text { Média } \\
\text { (DP) }\end{array}$ & $\begin{array}{c}\mathbf{p} \\
\text { valor }\end{array}$ & $\begin{array}{l}\text { Média } \\
\text { (DP) }\end{array}$ & $\begin{array}{c}p \\
\text { valor }\end{array}$ \\
\hline \multicolumn{11}{|c|}{ Idade } \\
\hline$<=30$ anos & $\begin{array}{c}71.15 \\
(13.66)\end{array}$ & \multirow{3}{*}{0,705} & $\begin{array}{c}70.40 \\
(10.19)\end{array}$ & & $\begin{array}{c}73.14 \\
(15.22)\end{array}$ & \multirow{3}{*}{0.108} & $\begin{array}{c}67.72 \\
(9.688)\end{array}$ & & $\begin{array}{c}72,22 \\
(15,63)\end{array}$ & \multirow{3}{*}{0,224} \\
\hline $\begin{array}{c}>30 \text { e }<40 \\
\text { anos }\end{array}$ & $\begin{array}{c}68.86 \\
(15.33)\end{array}$ & & $\begin{array}{c}64.96 \\
(13.60)\end{array}$ & 0.214 & $\begin{array}{c}67.30 \\
(18.37)\end{array}$ & & $\begin{array}{c}65.31 \\
(12.75)\end{array}$ & 0.561 & $\begin{array}{c}67,3 \\
(12,70)\end{array}$ & \\
\hline$>=40$ anos & $\begin{array}{c}72.04 \\
(19.77) \\
\end{array}$ & & $\begin{array}{c}65.44 \\
(14.53) \\
\end{array}$ & & $\begin{array}{c}63.54 \\
(17.54) \\
\end{array}$ & & $\begin{array}{l}64.025 \\
(16.14) \\
\end{array}$ & & $\begin{array}{c}64,84 \\
(20,68)\end{array}$ & \\
\hline \multicolumn{11}{|c|}{ Sexo } \\
\hline Feminino & $\begin{array}{c}69.51 \\
(16.69)\end{array}$ & \multirow{2}{*}{0.097} & $\begin{array}{c}66.85 \\
(12.95)\end{array}$ & \multirow{2}{*}{0.950} & $\begin{array}{c}68.05 \\
(17.91)\end{array}$ & \multirow{2}{*}{0.611} & $\begin{array}{c}65.24 \\
(13.50)\end{array}$ & & $\begin{array}{c}67.56 \\
(17.04)\end{array}$ & \multirow{2}{*}{0.599} \\
\hline Masculino & $\begin{array}{c}77.11 \\
(13.04)\end{array}$ & & $\begin{array}{c}67.09 \\
(16.10) \\
\end{array}$ & & $\begin{array}{c}65.47 \\
(15.27) \\
\end{array}$ & & $\begin{array}{c}67.92 \\
(11.24) \\
\end{array}$ & 0.470 & $\begin{array}{r}70.00 \\
(13.19) \\
\end{array}$ & \\
\hline \multicolumn{11}{|c|}{ Estado Civil } \\
\hline Solteiro & $\begin{array}{c}71.96 \\
(16.98)\end{array}$ & \multirow[b]{2}{*}{0.6180} & $\begin{array}{c}68.18 \\
(15.11)\end{array}$ & \multirow[b]{2}{*}{0.611} & $\begin{array}{c}70.83 \\
(19.81)\end{array}$ & \multirow[b]{2}{*}{0.234} & $\begin{array}{c}67.69 \\
(14.04)\end{array}$ & \multirow[b]{2}{*}{0.335} & $\begin{array}{c}69.64 \\
(16.12)\end{array}$ & \multirow[b]{2}{*}{0.527} \\
\hline Casado & $\begin{array}{c}70.15 \\
(16.70)\end{array}$ & & $\begin{array}{c}66.71 \\
(12.20)\end{array}$ & & $\begin{array}{c}66.22 \\
(16.31)\end{array}$ & & $\begin{array}{c}64.91 \\
(12.90)\end{array}$ & & $\begin{array}{c}67.41 \\
(17.13)\end{array}$ & \\
\hline
\end{tabular}


$\mathrm{Na}$ análise das variáveis demográficas em relação aos domínios e a QV global, também não foi verificada diferença significativa $(p>0,05)$ (tabela 1). No entanto, piores médias de QV global foram encontradas no grupo etário $\geq 40$ anos $(64,84)$; a QV de mulheres, em comparação a de homens, foi menor em todos os domínios, excetuando-se o das relações sociais (68.05); solteiros apresentaram maior QV global (69.64) e em todos os domínios, quando comparados aos indivíduos casados (QV global: 67.41).

\section{Discussão}

A QV no trabalho (QVT) é um conceito complexo, multidimensional, que foi definido de diferentes formas por diversos pesquisadores. A QVT se refere à satisfação do empregado com sua vida no trabalho. A melhora na QVT influencia positivamente na produtividade e procura por atualização profissional. Os resultados da QVT do enfermeiro influenciam na satisfação, estresse, burnout, satisfação do paciente e na qualidade do cuidado (FU et al., 2015). Diversos autores encontraram uma QV moderada entre profissionais enfermeiros (AKTER, 2017).

Em relação à faixa etária, foi verificada uma pior qualidade de vida global no grupo com mais de 40 anos (QVG=64,84). Outros autores corroboraram este achado (CRUZ et al., 2011; LAYTE et al., 2013). Para Fathimath (2014), a desvalorização profissional, o tratamento diferenciado entre médicos e enfermeiros, as escalas de trabalho inflexíveis, e a elevada carga de trabalho podem influenciar de forma negativa na QVT do enfermeiro. Yan et al. (2018) acrescentam a esta lista, a quantidade inadequada de enfermeiros, o trabalho laboral intenso, a pressão elevada, a baixa renda e o pouco suporte social.

Yan et al. (2018) demonstraram em seu trabalho uma correlação entre dores musculares e a prática laboral do enfermeiro, com prevalência próxima de $80 \%$, com maior risco conforme a idade. Layte et al. (2013) encontraram piora de QV em indivíduos com dor. Diante deste contexto, acredita-se que uma explicação para a piora da percepção da QV entre enfermeiros com maior idade, possa decorrer da presença de dores. Entretanto, a falta de controle da presença de dor no estudo, impossibilita esta confirmação. Para Queiroz e Souza (2012), os trabalhadores de enfermagem de maior idade podem apresentar maior dificuldade em manter relações sociais satisfatórias, devido às dificuldades acumuladas ao longo da vida, e ao cansaço físico e mental do dia a dia, influenciando em sua QV.

Em relação ao estado civil, chamou atenção uma melhora na QV entre solteiros, em todos os domínios, quando comparados com os enfermeiros casados. Este achado também foi encontrado por enfermeiros de hospitais norte-americanos (CRUZ et al., 2018) e do Brasil (QUEIROZ; SOUZA, 2012). A QV parece ter uma correlação positiva com os anos de estudo, renda mensal, anos de experiência e ambiente de trabalho (AKTER et al., 2017). Almaki et al. (2012) apontaram em seu trabalho, a dificuldade de equilíbrio entre o trabalho do enfermeiro e sua necessidade familiar, refletindo na QV. Sendo, portanto, uma explicação para a maior QV entre solteiros. Para Queiroz e Souza (2012), indivíduos casados possuem responsabilidades familiares, afetivas, econômicas e sociais, que tornam o trabalho mais desgastante, o que pode comprometer sua QV; enquanto os solteiros costumam direcionar suas preocupações mais frequentemente a projetos pessoais.

Ainda em relação à maior QV de solteiros, outra explicação seria a renda, uma vez que indivíduos casados comumente possuem dependentes, o que diminui sua renda percapita quando comparado aos solteiros. Dentro deste contexto, diversos trabalhos, encontraram uma correlação positiva entre QV e renda (OHAERI et al., 2008; CRUZ et al., 2011). Apesar das diversas explicações possíveis em relação ao estado civil, existem trabalhos que encontraram resultados diferentes (ALMAKI et al., 2012; MORADI et al., 2014). 
Encontrou-se também, no presente estudo, uma maior prevalência de mulheres $(84,85 \%)$. Para Queiroz e Souza, (2012), esta baixa prevalência de homens na profissão, pode ser vista como um problema, uma vez que a presença masculina é de extrema importância, devido às demandas físicas que os trabalhadores de enfermagem estão expostos, desempenhando, portando, um papel significativo nas práticas assistenciais de enfermagem.

No que concerne a QV, as mulheres apresentaram uma pior nota em todos os domínios, exceto quanto às relações sociais. Cruz et al. (2018), verificaram uma maior habilidade de enfrentamento proativo entre enfermeiros do sexo masculino e relacionaram a influência do enfrentamento proativo na QV, o qual se mostra associado a um bom controle interno, um enfrentamento ativo e a auto eficácia. Para Chang e Taylor (2013), o enfrentamento proativo está relacionado a uma baixa ocorrência de burnout entre enfermeiros. A pior QV atribuída ao sexo feminino vai ao encontro de achados de outros autores, os quais encontraram um maior risco de estresse em enfermeiras mulheres (MERLANI et al., 2011). Para Queiroz e Souza (2012), as atividades e responsabilidades profissionais e os afazeres domésticos podem contribuir para a menor QV das enfermeiras. Diversamente ao encontrado, Almaki (2012) atribuiu uma maior QVT atribuída ao sexo feminino, na Arábia Saudita.

No que diz respeito à QV nos diferentes níveis de atenção a saúde, não houve diferença significativa entre a APS e o HU, no entanto, houve uma maior diferença de médias no domínio físico (HU: 66,6 x APS: 73,7). O domínio físico é composto pelas facetas: dor e desconforto, energia e fadiga, sono e repouso, mobilidade, atividades da vida cotidiana, dependência de medicação ou de tratamentos, e capacidade de trabalho (GRUPO DE ESTUDOS EM QUALIDADE DE VIDA, 2016). O trabalho de Cruz et al. (2018), que também avaliou a QV na atenção terciária, nos Estados Unidos, o trabalho de Queiroz e Souza (2012), que avaliou os mesmos parâmetros no Brasil, e o trabalho de Silva et al. (2017), que avaliou profissionais da saúde no Brasil, também encontraram a pior média de QV no domínio físico.

Para Queiroz e Souza (2012) a baixa nota no domínio físico dos enfermeiros que atuam nos hospitais se deve aos desconfortos, tais como, dor, fadiga, sono, dependência de medicações e produtos cafeinados e ao comprometimento da mobilidade. Para Silva et al. (2017), os profissionais da enfermagem frequentemente apresentam dor nas costas e desordens musculoesqueléticas, devido a postura adotada durante a atividade laboral, e a fadiga decorrente de situações estressoras do ambiente de trabalho, tais como más condições de trabalho e falta de materiais. Estas dificuldades quando associadas ao trabalho em turnos, e ao acúmulo de mais de um emprego, levam ao cansaço e pouco descanso, causando dores, diminuição de energia e cansaço, influenciando na piora da QV.

Pode-se inferir que a melhor média de QV no domínio físico da APS esteja associada à diversidade do ambiente de trabalho, sugerindo ser este um local de atuação menos insalubre. Uma explicação seria a ausência de turnos, o trabalho diurno, o menor desgaste físico com deslocamento de pacientes, o menor contato com a dor, o sofrimento humano e a morte.

Para Schrader et al. (2012), o prazer com a atividade de cuidar, atribuída pelos enfermeiros da APS, e a sensação de utilidade trazem recompensa moral pelo esforço no trabalho, sendo um fator determinante da QVT. Outro fator determinante mencionado são as boas relações interpessoais estabelecidas, principalmente entre profissionais de saúde.

O domínio com pior média $(64,5)$ foi o meio ambiente dos enfermeiros do HU. Este domínio é composto pelas facetas: segurança física e proteção, ambiente no lar, recursos financeiros, cuidados de saúde e sociais: disponibilidade e qualidade, oportunidades de adquirir novas informações e habilidades, participação em, e oportunidades de 
recreação/lazer, ambiente físico, e transporte (GRUPO DE ESTUDOS EM QUALIDADE DE VIDA, 2016).

De modo geral, o meio ambiente vivenciado pelos enfermeiros do hospital é considerado insalubre e estressante (CHOI; LEE, 2017). Para Almaki (2012), a ausência de segurança no trabalho, a carência de equipamentos e insumos, o difícil equilíbrio entre trabalho e necessidades familiares, a escassez de recursos humanos, a falta de locais para descanso, e a pouca oportunidade de crescimento profissional, corroboram com uma pior QV. Choi et al. (2017) encontraram em seu trabalho uma prevalência de experiência de violência no trabalho no último ano de $95 \%$, entre enfermeiros que atuam em hospitais na Coreia, e uma relação direta com uma pior QVT e uma maior intenção de demissão.

Estudos apontam estratégias de melhoria da QV de enfermeiros, tais como, otimismo, pró-atividade (CRUZ et al., 2018), liderança, quantidade adequada de profissionais, tratamento igual entre diferentes profissões, escalas de trabalhos flexíveis, menor carga de trabalho (FATHIMATH et al., 2014), maior renda, suporte social (YAN et al., 2018), exercício físico, massagem, música, relaxamento e suporte de supervisores (SILVA et al., 2017).

\section{Considerações finais}

Apesar de não haver diferença significativa entre a QV dos enfermeiros nos diferentes níveis de atenção à saúde, o presente estudo possibilitou analisar e conhecer os pontos de fragilidade na QV destes profissionais e fomentou questões importantes para a compreensão do contexto de trabalho do enfermeiro. Acredita-se que este conhecimento possibilitará o planejamento de medidas de melhoria da QV de enfermeiros, podendo impactar positivamente na gestão dos serviços de saúde e na qualidade da assistência prestada. A limitação deste trabalho decorreu da ausência de controle dos fatores de confusão como: tempo de serviço, burnout, estresse, dor, renda, tipo de vínculo, experiência de violência, setor de atuação, entre outros. Novos estudos, com os fatores de confusão e uma amostra ampliada se fazem necessários, bem como a investigação de serviços privados, e o estudo de outras regiões do país, podendo encontrar resultados diversos.

\section{Referências}

AKTER, N. et al. Factors predicting quality of work life among nurses in tertiary-level hospitals, Bangladesh. International Nursing Review, p. 1-8, 2017. Disponível em: <https://www.ncbi.nlm.nih.gov/pubmed/29105085> Acesso em: 06 mar. 2020.

ALMALKI, M. J.; FITZGERALD, G.; CLARK, M. Quality of work life among primary health care nurses in the Jazan region, Saudi Arabia: A cross-sectional study. Human Resources for Health, v. 10, n. 1, p. 1, 2012. Human Resources for Health. Disponível em: $\quad<$ https://human-resources-health.biomedcentral.com/articles/10.1186/1478-4491-1030> Acesso em: 06 mar. 2020.

BATTU, N.; CHAKRAVARTHY, K. Quality of work life of nurses and paramedical staff in hospitals. International Journal of Business and Administration Research Review, v. 2, n. 2347, p. 200-207, 2014. Disponível em: <http://ijbarr.com/downloads/2014/vol2issue4/25.pdf> Acesso em: 06 mar. 2020.

BECK, C. L. C.; STEKEL, L. M. C.; GONZALES, R. M. B.; DONADUZZI, J. C. O trabalho da enfermagem em unidades críticas e sua repercussão sobre a saúde dos trabalhadores. 
Escola Anna Nery Revista de Enfermagem, v. 10, n. 2, p. 221-7, 2006. $\square$ Disponível em: <http://www.scielo.br/scielo.php?script=sci arttext\&pid=S1414-81452006000200008> Acesso em: 06 mar. 2020.

BROOKS, B. A.; ANDERSON, M. A. Defining quality of nursing work life. Nursing Economics, v.23, n.6, 319-26, 2005. Disponível em: <https://www.ncbi.nlm.nih.gov/pubmed/16459904> Acesso em: 06 mar. 2020.

BROOKS, B. A. et al. Assessing the Quality of Nursing Work Life. Nursing Administration Quarterly, v. 31, n. 2, p. 152-157, 2007. Disponível em: <https://www.ncbi.nlm.nih.gov/pubmed/17413509> Acesso em: 06 mar. 2020.

CHANG, K.; TAYLOR, J. Do your employees use the right stress coping strategies. International Journal of Commerce and Strategy, v. 5, n. 2, p. 099-116, 2013. Disponível em: <http://usir.salford.ac.uk/id/eprint/31164/1/IJCS.pdf> Acesso em: 06 mar. 2020.

CHOI, S. H.; LEE, H. Workplace violence against nurses in Korea and its impact on professional quality of life and turnover intention. Journal of Nursing Management, v. 25, n. 7, p. 508-518, 2017. Disponível em: <https://www.ncbi.nlm.nih.gov/pubmed/28547784> Acesso em: 06 mar. 2020.

CRUZ, J. P. et al. Optimism, Proactive Coping and Quality of Life among Nurses: A CrossSectional Study. Journal of Clinical Nursing, v.27, n.9-10, p.2098-2108, 2018. Disponível em: <https://www.ncbi.nlm.nih.gov/pubmed/29603804> Acesso em: 06 mar. 2020.

CRUZ, L. N. et al. Quality of life in Brazil : normative values for the Whoqol-bref in a southern general population sample. Quality Life Research, v. 20, p. 1123-1129, 2011. Disponível em: <https://www.ncbi.nlm.nih.gov/pubmed/21279448> Acesso em: 06 mar. 2020.

FATHIMATH, S.; CHONTAWAN, R.; CHITPAKDEE, B. Quality of Work Life and Job Performance among Nurses in the Tertiary Care Hospital, Maldives. Nursing Journal, v.41, n.3, p.105-118, 2014. Disponível em: <http://newtdc.thailis.or.th/docview.aspx?tdcid=123761> Acesso em: 06 mar. 2020.

FLECK, M. P. DE A. O instrumento de avaliação de qualidade de vida da Organização Mundial da Saúde (WHOQOL-100): características e perspectivas. Ciência \& Saúde Coletiva, v.5, n.1, p. 33-38, 2000. Disponível em: $<$ http://www.scielo.br/scielo.php?pid=S1413$81232000000100004 \&$ script $=$ sci abstract\&tlng $=p t>$

$\mathrm{FU}, \mathrm{X}$. et al. Validation of the Chinese version of the quality of nursing work life scale. PLoS ONE, v. 10, n. 5, p. 1-12, 2015. Disponível em: $<$ https://journals.plos.org/plosone/article?id=10.1371/journal.pone.0121150> Acesso em: 06 mar. 2020.

GRAPHPAD SOFTWARE, 2018. San Diego, Califórnia, USA. Disponível em: <www.graphpad.com>Acesso em: 06 mai. 2018. 
GRUPO DE ESTUDOS EM QUALIDADE DE VIDA, 2016. Universidade Federal do Rio Grande do Sul. Rio Grande do Sul, Brasil. Disponível em:<https://www.ufrgs.br/qualidep/qualidade-de-vida/projeto-whogol-bref>Acesso em:13 out. 2018.

HOLMES, E. S.; FARIAS, J. A.; SOUSA, M. B. Síndrome de Burnout em enfermeiros na atenção básica: repercussão na qualidade de vida. Revista de Pesquisa Cuidado é Fundamental Online, v. 6, n. 4, p. 1384-1395, 2014. Disponível em: <https://pesquisa.bvsalud.org/portal/resource/pt/bde-25816> Acesso em: 06 mar. 2020.

JANG, I.; KIM, Y.; KIM, K. Professionalism and professional quality of life for oncology nurses. Journal of Clinical Nursing, p. 2835-2845, 2016. Disponível em: <https://www.ncbi.nlm.nih.gov/pubmed/27335236> Acesso em: 06 mar. 2020.

KABAD, L. Qualidade de vida dos funcionários administrativos de uma universidade privada. 2011. 112f. Dissertação (Mestrado em Psicologia) - Universidade Católica Dom Bosco, Campo Grande, MS, 2011. Disponível em: <https://site.ucdb.br/public/mddissertacoes/8161-qualidade-de-vida-dos-funcionarios-administrativos-de-umauniversidade-privada.pdf>. Acesso em: 12 out. 2018.

LAYTE, R.; SEXTON, E.; SAVVA, G. Study. Quality of Life in Older Age: Evidence from an Irish Cohort Study. Journal of the American Geriatrics Society, v.61, p. 299-305, 2013. Disponível em: <https://www.ncbi.nlm.nih.gov/pubmed/23662722> Acesso em: 06 mar. 2020.

MERLANI, P. et al. Burnout in ICU Caregivers: A Multicenter Study of Factors Associated to Centers. American Journal of Respiratory and Critical Care Medicine, v. 184, n. 13, p. 1140-1146, 2011. Disponível em: <https://www.ncbi.nlm.nih.gov/pubmed/21852543> Acesso em: 06 mar. 2020.

MORADI, T.; MAGHAMINEJAD, F.; AZIZI-FINI, I. Quality of Working Life of Nurses and its Related Factors. Nursing and midwifery studies, v. 3, n. 2, p.1-6, 2014. Disponível em: <https://www.ncbi.nlm.nih.gov/pmc/articles/PMC4228533/> Acesso em: 06 mar. 2020.

MOSADEGHRAD, A. M.; FERLIE, E.; ROSENBERG, D. A study of relationship between job stress, quality of working life and turnover intention among hospital employees. Health Services Management Research, v.24, p.170-181, 2011. Disponível em: $<$ https://journals.sagepub.com/doi/abs/10.1258/hsmr.2011.011009> Acesso em: 06 mar. 2020 .

OHAERI, J. U.; AWADALLA, ÆE. A. W.; GADO, ÆE. O. M. Subjective quality of life in a nationwide sample of Kuwaiti subjects using the short version of the WHO quality of life instrument. Social Psychitry Epidemio, v.44, p.693-701, 2009. Disponível em: <https://ink.springer.com/article/10.1007/s00127-008-0477-z> Acesso em: 06 mar. 2020.

PEDROSO, B; PILATTI, L.A.; DOS REIS, D.R. Cálculo dos escores e estatística descritiva do WHOQOL-100 utilizando o Microsoft Excel. Revista Brasileira de Qualidade de Vida, v. $01, \quad$ n. $01, \quad$ p. 23-32, 2009. Disponível em: $<$ https://pdfs.semanticscholar.org/4bd1/8f10d61a7941f0f88225ba8ee51ea743aeb0.pdf> Acesso em: 06 mar. 2020. 
PERRY, L. et al. Health, workforce characteristics, quality of life and intention to leave: The 'Fit for the Future' survey of Australian nurses and midwives. Journal of Advanced Nursing, v. $73, \quad$ n. 11, p. 2745-2756, 2017. Disponível em: $<$ https://onlinelibrary.wiley.com/doi/full/10.1111/ian.13347> Acesso em: 06 mar. 2020.

QUEIROZ, D. L.; SOUZA, J. C. Qualidade de vida e capacidade para o trabalho de profissionais de enfermagem. Psicólogo Informação, n.16, p103-126, 2012. Disponível em: $<$ https://www.metodista.br/revistas/revistasmetodista/index.php/PINFOR/article/view/3999 > Acesso em: 06 mar. 2020.

ROSSI, S.; SANTOS, G.; PASSOS, P. A síndrome de burnout no enfermeiro: um estudo comparativo entre atenção básica e setores fechados hospitalares. Revista de Pesquisa Cuidado é Fundamental Online, v.2, n.4, p.1232-1239, 2010. Disponível em: $<$ https://www.redalyc.org/pdf/5057/505750833002.pdf> Acesso em: 06 mar. 2020.

SCHRADER, G. et al. Trabalho na Unidade Básica de Saúde: implicações para a qualidade de vida dos enfermeiros. Revista Brasileira de Enfermagem, v. 65, n. 2, p. 222-228, 2012. Disponível em: <http://www.scielo.br/scielo.php?pid=S003471672012000200004\&script=sci arttext> Acesso em: 06 mar. 2020.

SILVA, B. K. R. et al. Correlation Between Stress and Quality of Life Experienced by Caregivers: Perception of a Group of Healthcare Professionals. The Open Nursing Journal, v. 11, n. 1, p. 135-141, 2017. Disponível em: <http://benthamopen.com/FULLTEXT/TONURSJ-11-135>. Acesso em: 06 mar. 2020.

STEPHENS, Grover C. Our Quality of Life Arouses Student Skeptics. Science, Illinois, v. 166, n. 3902, p. 167-168, out. 1969.

SUN, N. et al. The correlation between quality of life and social support in female nurses. Journal of Clinical Nursing, v. 26, n. 7-8, p. 1005-1010, 2017. Disponível em: <http://doi.wiley.com/10.1111/jocn.13393>.Acesso em: 06 mar. 2020.

WHOQOL GROUP, T. The world health organization quality of life assessment (whoqol): position paper from the world health organization. Social Science \& Medicine, v. 41, n. 10, p.1403-1409, $1995 . \quad$ Disponível em: <https://www.sciencedirect.com/science/article/pii/027795369500112K> Acesso em: 06 mar. 2020.

YAN, P. et al. Correlation analysis between work-related musculoskeletal disorders and the nursing practice environment, quality of life, and social support in the nursing professionals. Medicine, v. 97, n. 9, p. e0026, 2018. Disponível em: $<$ http://www.ncbi.nlm.nih.gov/pubmed/29489648\%0Ahttp://www.pubmedcentral.nih.gov/art iclerender.fcgi?artid=PMC5851758>. Acesso em: 06 mar. 2020. 